\title{
Effect ofFriction onthe Cutting Forces in High Speed Orthogonal Turning of Al 6061-T6
}

\author{
Dr. H. D. Gopalakrishna ${ }^{1}$, Jayghosh S. Rao ${ }^{1}$, Shruthi N. Kumar ${ }^{1}$, Vishwajith V. \\ Shetty ${ }^{1}$, Karthik Rai ${ }^{1}$

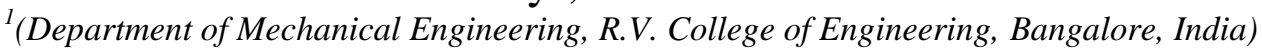

\begin{abstract}
In this work, the effect of coulomb friction on the cutting forces generated during the orthogonal turning of Aluminium 6061-T6 is studied by a parametric variation of friction co-efficient. The simulations were carried out in the commercial software ANSYS using the AUTODYN explicit solver. The finite element model assumed plane strain condition to be active during machining so that computation could be performed at reduced cost. Steinberg-Cochran-Guinan-Lund material model was used to provide flow stress data for the plastic deformation during chip formation. Cutting force and thrust force values were obtained for varied values of co-efficient of friction.
\end{abstract}

Keywords: Al6061-T6, cutting forces, friction co-efficient, Lagrangian mesh, metal cutting

\section{Introduction}

Metal cutting is one of the most used production processes in the industry. In modern manufacturing, there has been a keen interest in high-efficiency machining. This has not only brought about a change in the machining processes used, but also paved way for new methods and techniques to analyse these processes. Cost and time limitations along with technological inadequacy have deterred the use of experiments in analysing the metal cutting processes thoroughly. Also,dataobtained during machining a given material becomes obsolete due to the continuous improvement in the material database. In such scenarios computer simulations of the cutting process can potentially reduce the number of iterations in the design of experiments and result in substantial cost savings.

The development of more lightweight parts which must nevertheless meet unchanged strength requirements has given rise to an ever-growing use of aluminium alloys. Particularly Al 6061-T6 finds its applications in construction of aircraft structures, automobile parts, bicycle frames etc.

A finite element model using Lagrangian meshing for orthogonal high speed machining was developed by Marusich and Ortiz [1]. Continuous re-meshing and adaptive meshing, mesh on mesh contact with friction, and full thermo mechanical coupling were the distinct features of this model.

TugrulÖzel and TaylanAltan[2]established a methodology to determine flow stress at high deformation rates and temperatures that are encountered and the friction at the tool chip interface simultaneously.ZouharandPiska[3] explored the finite element modeling of orthogonal machining process using tools with different geometries utilizing ANSYS/LS-DYNA.

In any machining process, friction plays a critical role. It affects temperatures, cutting forces, tool wear, and surface roughness. The frictional force is controlled by the co-efficient of friction between the two materials in relative motion. The study of the effect of frictional co-efficient on the output variables is of utmost importance. A. Moufki et al[4] studied the effect of cutting conditions and material behaviour on the mean friction using an analytical thermo-mechanical model. T. Ozel[5] researched the effects of interfacial friction models on finite elementsimulations.L. Filice et al[6]investigated the role of different friction models within a 2D simulation of orthogonal cutting.Arrazola and Ozel[7] investigated the influence of limiting shear stress at the tool chip contact on the frictional conditions.

he current work deals with the high speed orthogonal turning of Al 6061-T6 under plane strain conditions. The objective of the present work is to track the variation of cutting forces under the influence of different values of co-efficient of friction. Lagrangian mesh formulation is used in this model due to its inherent benefits over the Eulerian formulation while calculating variable values at the free surface. The Steinberg-CochranGuinan-Lund constitutive model is opted for over the Johnson-Cook model. The simulations are conducted in ANSYS using the explicit dynamics solver, AUTODYN. 


\subsection{Geometry and Meshing}

\section{Finite Element Model}

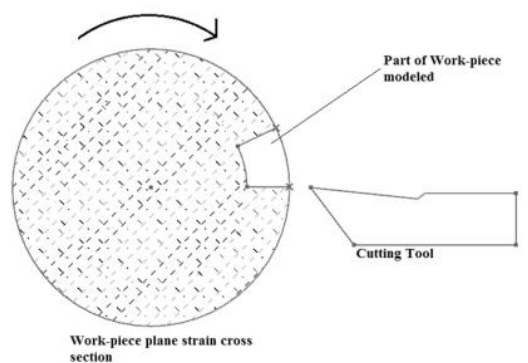

Figure 1: Plane strain cross section of cylindrical work-piece

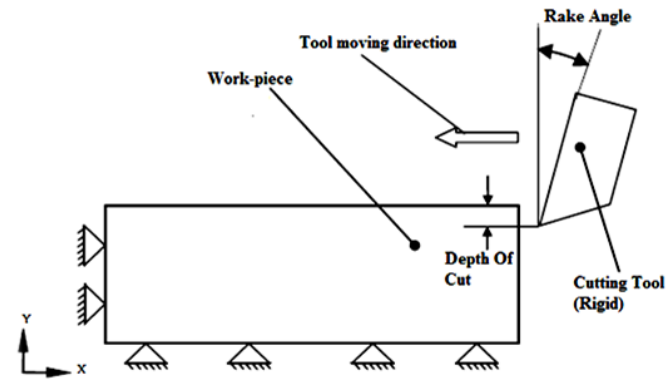

Figure 2: Geometry and constraints used in FE model

In this present study it is assumed that the turning process is completely orthogonal and that the condition of plane strain is active in the orthogonal plane during the operation.This is shown in Fig. 1. A small strip of the work-piece is modelled as shown in Fig. 2 for the present analysis.

The tool is modelled to be rigid, and the work-piece deformable. The tool has been modelled with a rake of $+6^{\circ}$, nose radius of $0.2 \mathrm{~mm}$ and an arbitrarily large clearance angle. The work-piece dimensionis $(7 \times 2) \mathrm{mm}$.

The cutting speed chosen is $30 \mathrm{~m} / \mathrm{s}$ as it is the maximum cutting speed possible for the high speed machining of Aluminium alloys[8]. Depth of cut is fixed to $0.5 \mathrm{~mm}$. All the stated parameters were constant. The friction coefficient is varied from 0 to 1 in steps of 0.1 .

Lagrangian mesh formulation is used in all simulations. A dense mesh is used in the region of cut to improve resolution of chip morphology. The finite element model consisted of 15123 nodes and 14835 elements. The smallest elements had an edge length of $1.8 \times 10^{-2} \mathrm{~mm}$. The element chosen is PLANE182. The meshed model is depicted in Fig. 3.

The penalty approach is used to model the contact behaviour between the tool and work-piece. This algorithm is used since it provides the best results without any element penetration that is otherwise encountered in problems of this kind.

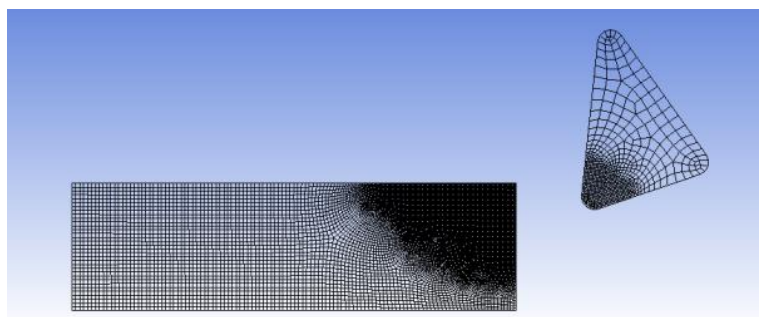

Figure 3: Finite Element Geometry and Mesh for the work-piece and tool

\subsection{Friction Model}

Coulomb's friction model theorises that frictional force exists due to interfacial bonds that are created at the true areas of contact between two surfaces. To achieve relative motion, these bonds must be sheared. The mathematical description of coulomb friction used by the solver isgiven in equation (1):

$F=\mu N($ or $) \quad \tau=\mu \sigma$

The frictional force is independent of the apparent area of contact between the surfaces. No surface is completely smooth. At a microscopic level, there exist peaks and craters on every surface as shown in Fig. 4. When two surfaces are in contact, the top surface rests due to the support provided from these peaks. The peaks 
deform plastically even due to the self-weight of the body since pressure at these peaks is well beyond yield pressure. In this process, the true area of contact increases so that the required reaction force can be generated. When the load is increased, there is more plastic deformation and hence more area of contact. Hence the frictional force is directly related to the normal reaction between the surfaces in contact.

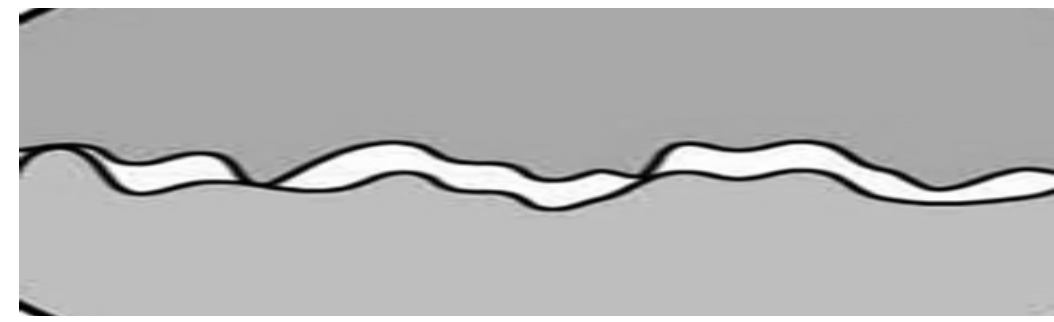

Figure 4: Microscopic view of two surfaces in contact

\subsection{Material Model/ Flow stress Model and Chip formation}

In the modelling of metal cutting process, generally, the Johnson-Cook (JC) material model has been used throughout. In this work, Steinberg-Cochran-Guinan-Lund Model is selected for its reliability in high strain conditions.[9]

Particularly in high speed machining, extremely high strain rates $\left(1.67 \times 10^{5} \mathrm{sec}^{-1}\right.$ at $500 \mathrm{~m} / \mathrm{min}$ cutting speed and $0.05 \mathrm{~mm}$ feed) and temperatures $\left(1400^{\circ} \mathrm{C}\right)$ at the chip-tool interface occur in the primary deformation zone and secondary deformation zone [2].

Since the model incorporated a Lagrangian mesh, erosion was a suitable technique to simulate the chip formation in the finite element model. The criterion for erosion to activate was the point of material failure based on the Steinberg-Cochran-Guinan-Lund material constitutive model.

The flow stress in this model is given by equations (2) to (5):[9].

$\sigma_{y}\left(\varepsilon_{p}, \dot{\varepsilon}_{p}, T\right)=\left[\sigma_{a} f\left(\varepsilon_{p}\right)+\sigma_{t}\left(\dot{\varepsilon}_{p}, T\right)\right] \mu(p, T) / \mu_{0} ; \sigma_{a} f \leq \sigma_{\max }$ and $\sigma_{t} \leq \sigma_{p}$

where the strain hardening function $(f)$ has the form

$f\left(\varepsilon_{p}\right)=\left[1+\beta\left(\varepsilon_{p}+\varepsilon_{p i}\right)\right]^{n}$

$\dot{\varepsilon}_{p}=\left[\exp \left[2 U_{k}\left(1-\sigma_{t} / \sigma_{p}\right)^{2} / k_{b} T\right] / C_{1}+C_{2} / \sigma^{-1}\right.$

$\sigma_{t} \leq \sigma_{p}$

The constants $C_{1}, C_{2}$ are given by the relations

$C_{1}=\rho_{d} L_{d} a b^{2} v / 2 w^{2} ; C_{2}=D / \rho_{d} b^{2}$

The work piece material properties areindicated in TABLE1.

Table 1: Work piece Material Properties

\begin{tabular}{clcc}
\hline Sl. No. & Property & Value & Units \\
\hline $\mathbf{1}$ & Density & 2703 & $\mathrm{~kg} / \mathrm{m} 3$ \\
$\mathbf{2}$ & Specific Heat & 885 & $\mathrm{~J} / \mathrm{kg} / \mathrm{K}$ \\
$\mathbf{3}$ & Steinberg-Guinan Strength & & \\
& Initial Yield Stress & $2.90 \mathrm{E}+08$ & $\mathrm{~Pa}$ \\
& Maximum Yield Stress & $6.80 \mathrm{E}+08$ & $\mathrm{~Pa}$ \\
& Hardening Constant & 125 & \\
& Hardening Exponent & 0.1 & \\
& Derivative dG/dP G'P & 1.8 & $\mathrm{~Pa} /{ }^{\circ} \mathrm{C}$ \\
& Derivative dG/dT G'T & $-1.70 \mathrm{E}+07$ & \\
& Derivative dY/dP Y'P & 0.018908 & ${ }^{\circ}$ \\
& Melting Temperature & 946.85 & $\mathrm{~Pa}$ \\
\hline
\end{tabular}

\section{Results And Discussions}

The simulations showed that cutting force and thrust force varied in exactly the same manner with respect to perturbations in the co-efficient of friction.The cutting force washigher than the thrust force at all times. The forces increased as the co-efficient of friction increased till the value of 0.5 , after which the forces reduced in magnitude and remained almost constant even with increase in co-efficient of friction. This has been indicated in TABLE2 and Fig. 5. 
Table 2: Coefficient of Friction, Average Cutting and Thrust forces

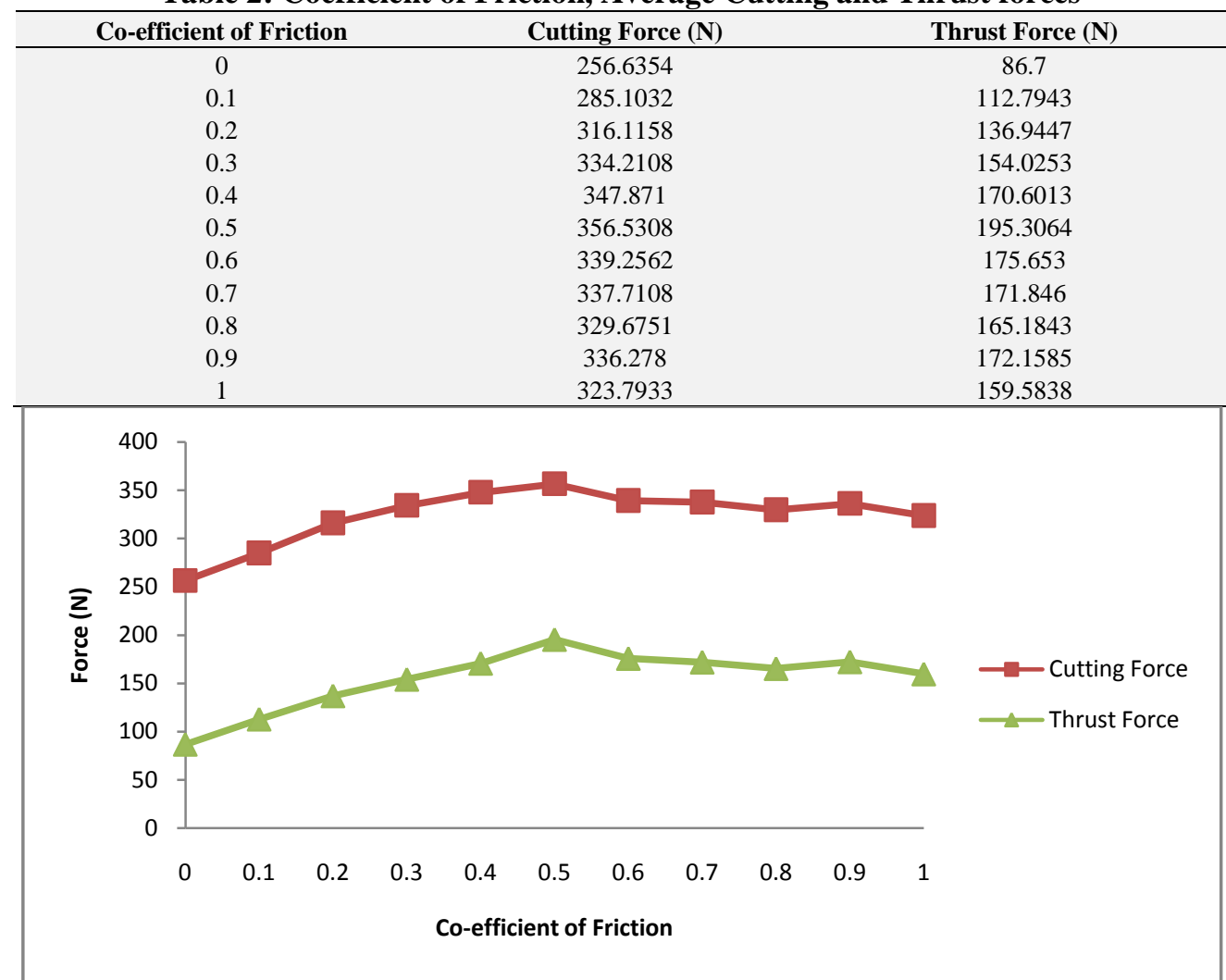

Figure 5:Variation of Average Cutting force and Thrust force v/s Co-efficient of Friction

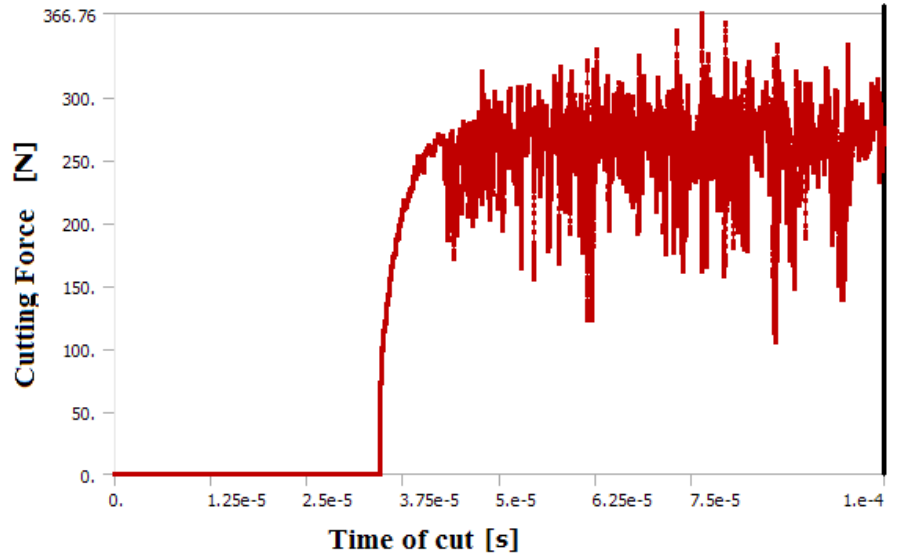

Figure 6: Variation of cutting force with time of cut for a zero friction case

Fig. 6 shows the variation of cutting force with time of cut during one particular simulation conducted. There is a steep increase in the cutting force between the point of first contact and the point of shear of the work-piece to form the chip. After the chip has been formed, there are high frequency oscillations in cutting force. This is due to the element erosion that is active during the analysis. As the tool moves through the work-piece, it compresses the elements in the shear zone. These elements deform and resist the cutting action of the tool. Some elements are compressed to the material failure limit and are deleted. This deletion is sudden and accounts for the drop in magnitude of cutting force. The tool then proceeds to compress the next element and the process repeats.

Also observed was the decrease in chip curl as the co-efficient of friction was increased. This is due to the resistance to sliding on the tool rake face due to the higher value of frictional force. Furthermore, in order to accommodate the flow of the chip in spite of the resisting friction, the chip thickness increases. These phenomena are depicted in Fig. 7. 


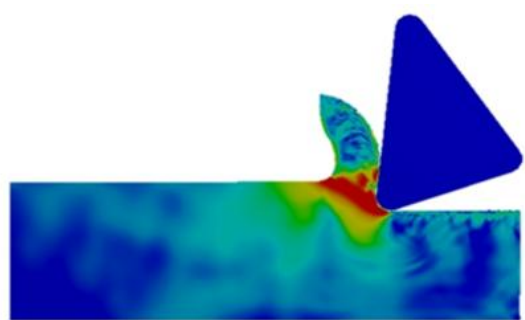

(a)

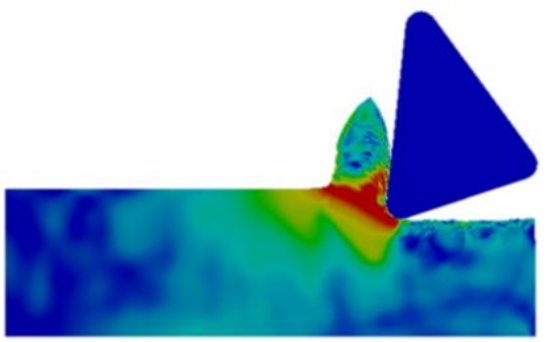

(c)

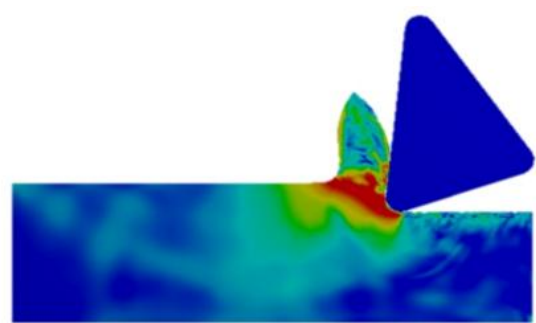

(b)

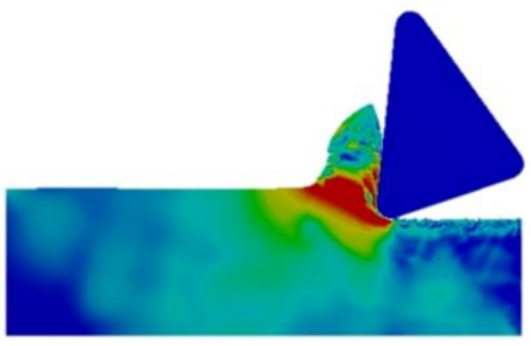

(d)

Figure 7: Variation in chip curl and chip thicknesses for values of co-efficient of friction (a) 0 (b) 0.1 (c) 0.2 (d) 0.3

In light of the results obtained, it is clearly evident that the forces generated during turning remain low when the friction co-efficient has a value below 0.5. Due to this, the power consumption and cutting temperatures are reduced while the surface finish is improved.

\section{Conclusion}

The variation of cutting forces and thrust forces with the parametric variation of coefficient of friction during the orthogonal turning of Al 6061-T6 is successfully determined by conducting a number of finite element simulations. The analysis is carried out in the ANSYS software using the AUTODYN explicit solver assuming plain strain condition. High frequency oscillations are observed in the measurement of cutting forces which is justified. It is found that the forces are low when the value of friction coefficient is below 0.5.In order to reduce the co-efficient of friction, coated tool inserts in which the coated material has low co-efficient of friction relative to the work-piece could be used. Alternatively, lubricants may be used.

\section{Nomenclature}

$\mathrm{F}, \tau=$ Frictional force acting at the chip rake face interface

$\mu=$ Co-efficient of friction

$\mathrm{N}, \sigma=$ Reaction force

$\varepsilon_{p}=$ Initial equivalent plastic strain

$\dot{\varepsilon}_{p}=$ Plastic strain-rate

$\mathrm{T}=$ Reference temperature

$\sigma_{a}=$ Athermal component of the flow stress

$f\left(\varepsilon_{p}\right)=$ Function that represents strain hardening

$\sigma_{t}=$ Thermally activated component of the flow stress

$\mu(p, T)=$ Pressure- and temperature-dependent shear modulus

$\mu_{0}=$ Shear modulus at standard temperature and pressure

$\sigma_{\max }=$ Saturation value of the athermal stress

$\sigma_{p}=$ Saturation of the thermally activated stress $=$ Peierls stress

$\beta, n=$ Work hardening parameters

$L_{d}=$ Length of a dislocation segment

$2 U_{k}=$ Energy to form a kink-pair in a dislocation segment of length $L_{d}$

$k_{b}=$ Boltzmann constant

$\rho_{d}=$ Dislocation density

$a=$ Distance between Peierls valleys

$b=$ Magnitude of the Burgers' vector

$v=$ Debye frequency

$w=$ Width of a kink loop

$D=$ Drag coefficient. 


\section{References}

[1] T. D. Marusich And M. Ortiz,Modelling And simulation of high speed machining.International Journal for numerical methods in Engineering,38,1995,3675-3694.

[2] TugrulOzelandTaylanAltan, Modelling of high speed machining processes for predicting tool forces, stresses and temperatures using FEM simulations, CIRP International Workshop on Modelling of Machining Operations, 1998, 225-234.

[3] J. Zouhar, M. Piska, Modelling the orthogonal machining process using cutting tools with different geometry, MM Science Journal,2008, 48-51.

[4] Moufki, A. Molinari, D. Dudzinski, Modelling Of Orthogonal Cutting with a Temperature Dependent Friction Law, Journal of Mechanics and Physics of Solids,46,1998,2103-2138.

[5] TugrulOzel, Influence of Friction Models On Finite Element Simulations of Machining, International Journal of Machine Tools \& Manufacture,46, 2006, 518-530.

[6] L. Filice, F. Micari, S. Rizzuti, D. Umbrello, A Critical Analysis On The Friction Modelling In Orthogonal Machining, International Journal of Machine Tools \& Manufacture,47, 2007,709-714.

[7] PedroJ.Arrazola, TugrulOzel, Investigations on the effects of friction modelling in finite element simulation of machining, International Journal of Mechanical Sciences, 52, 2010,31-42.

[8] H.M.Somashekar, N.LakshmanaSwamy, Chandrasekhar B., Effective Method to Measure Cutting Forces during High Speed Machining of Aluminium, International Journal Of Advanced Engineering Sciences And Technologies, 6, 2011,193-199.

[9] Biswajit Banerjee, An evaluation of plastic flow stress models for the simulation of high-temperature and high-strain-rate deformation of metals, University of Utah, 2008. 